\title{
Sarcopenia in older black South African women and relationships with physical activity and protein intake
}

\author{
Amy E. Mendham $^{1,2}$, Naomi E. Brooks ${ }^{3}$, Lisa K. Micklesfield ${ }^{4}$, Mieke Faber ${ }^{2}$, \\ Dirk L. Christensen ${ }^{5}$, Iain J. Gallagher ${ }^{3}$, Lillemor Lundin-Olsson ${ }^{6}$, Kathryn H. Myburgh ${ }^{7}$, \\ Sebastiana Kalula ${ }^{1}$, Feyisayo Odunitan-Wayas ${ }^{1}$ and Estelle V Lambert ${ }^{1}$ \\ ${ }^{1}$ University of Cape Town, Cape Town, South Africa, \\ ${ }^{2}$ South African Medical Research Coucil, Cape Town, South Africa, \\ ${ }^{3}$ University of Stirling, Stirling, United Kingdom, \\ ${ }^{4}$ Witwatersrand University, Johannesburg, South Africa, \\ ${ }^{5}$ University of Copenhagen, Copenhagen, Denmark, \\ ${ }^{6}$ Umea University, Umea, Sweden and \\ ${ }^{7}$ Stellenbosch University, Cape Town, South Africa
}

\begin{abstract}
Introduction: South Africa (SA) is a developing country with an ageing population. Adequate nutrition and physical activity (PA) protect against the loss of muscle mass and physical function, both of which are important components of sarcopenia. This study aimed to measure the prevalence of sarcopenia in older black SA women and investigate its associations with PA and protein intake.

Materials and Methods: Older black SA women (age, 68 (range; 60-85 years) $n=122$ ) completed sociodemographic questionnaires, $24 \mathrm{~h}$ urine collection (estimate protein intake), venous blood (hs-C-reactive protein (hs-CRP) and ferritin), functional tests (grip strength, 3 m timed-up-and-go (TUG), $10 \mathrm{~m}$ walk test) and PA monitoring (activPAL). Dual-energy x-ray absorptiometry whole-body scans assessed fat and fat-free soft tissue mass (FFSTM).

Results: According to the European Working group on Sarcopenia in Older People (EWGSOP)2, 2.5\% $(\mathrm{n}=3)$ had confirmed sarcopenia of a low severity based on normal physical function. Of the total cohort, $9 \%(\mathrm{n}=11)$ had low grip strength, $22.1 \%(\mathrm{n}=27)$ had a low appendicular skeletal muscle index (ASMI), and no women had low TUG (s) or gait speed (m/s). Higher ASMI was associated with lower hs-CRP $(p=0.05 ;$ Rho $=-0.209)$ and higher ferritin $(R h o=0.252 ; p=0.019)$, grip strength $(\mathrm{kg}, \mathrm{Rho}=0.223 ; \mathrm{p}=0.015)$, and gait speed $(\mathrm{m} / \mathrm{s}, \mathrm{Rho}=0.180 ; \mathrm{p}=0.050)$. Protein intake suggested intake of $41.8 \mathrm{~g} / \mathrm{day} / \mathrm{or} 0.51 \mathrm{~g} / \mathrm{kg}$ of body mass $/ \mathrm{day}$. Higher total protein intake $(\mathrm{g} / 24 \mathrm{~h})$, was associated with higher FFSTM $(\mathrm{kg})$ and ASMI $(\mathrm{p}<0.001)$. PA outcomes were not correlated with FFSTM or ASMI ( $\mathrm{p}>0.05)$, however, there was a strong positive correlation of TUG (s) and gait speed (m/s) with time spent: 1$)$ stepping per day (min) and; 2) at a high cadence (>100 steps/min) (all p <0.01). Daily step count was $7137 \pm 3233$ (mean \pm Standard deviation), with $97.9 \pm 38.7$ min of total time spent stepping and $12.6 \pm 16.8$ min spent stepping at a high cadence $(>100$ steps $/ \mathrm{min})$. Of note, $13.9 \%(\mathrm{n}=17)$ of women were completing $>10,000$ steps/day.
\end{abstract}

Discussion: Based on the EWGSOP2 criteria, there is a low prevalence of sarcopenia in older black SA women, explained by the maintenance of strength and physical function that directly related to PA, especially that performed at higher intensities. In contrast, low muscle mass was relatively prevalent $(22.1 \%)$ and was associated with low dietary protein and not PA. Notably, it may be important to review the cut-points of EWGSOP2 criteria to be specific to the older SA women from disadvantaged communities.

\section{Conflict of Interest}

There is no conflict of interest. 